\title{
Cone-rod dystrophy and amelogenesis imperfecta (Jalili syndrome): phenotypes and environs
}

Eye (2010) 24, 1734-1735; doi:10.1038/eye.2010.144

Correction to: Eye (2010) 24, 1659-1668; doi:10.1038/eye.2010.103; published online 13 August 2010

The author of the above article noted errors in the publication of this paper (AOP and in this issue).

In the second paragraph of the Discussion, the fourth sentence should read: The suspected founder of the genetic mutation in Gaza A was the fourth grandson of a man who had originally moved from Mekka in Hijaz (present
IK Jalili

day Saudi Arabia) and settled in Salama village near Jaffa, now Kafr Shalem, marrying into the indigenous population.

In Retinal phenotypes in the Discussion, the last sentence should read: Sibling C phenotype is that of type A with colobomatous macular lesion and peripheral pigmentary disturbances present at a much earlier age of 17 years (Figure 2l) than his counterpart with colobomatous lesion in Gaza A, who was 50 years of age ${ }^{37}$ (unpublished data).

Figure 2 and its legend were also incorrect. The correct figure and legend are shown below.

The author would like to apologize for these errors.
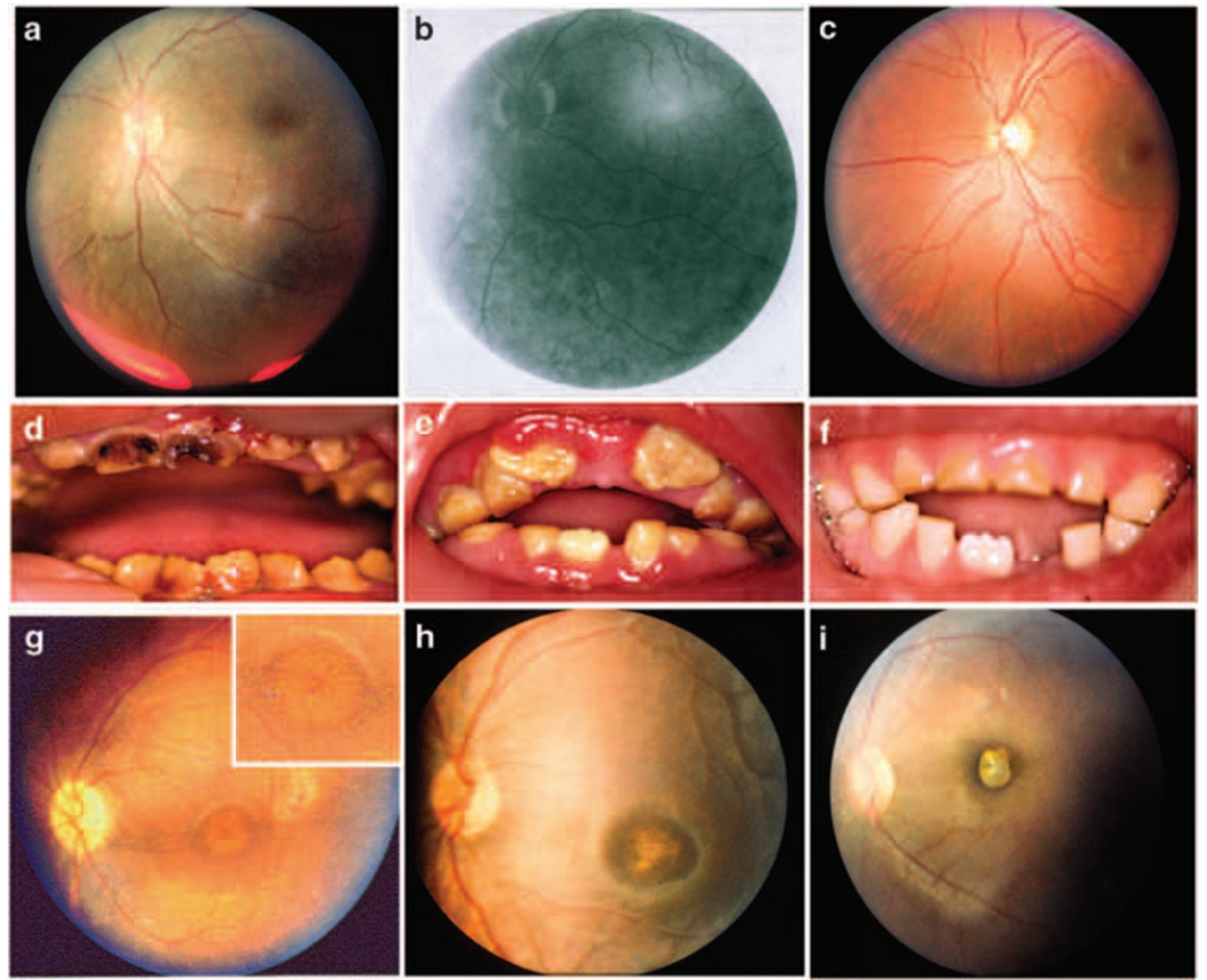

Figure 2 (continued) 

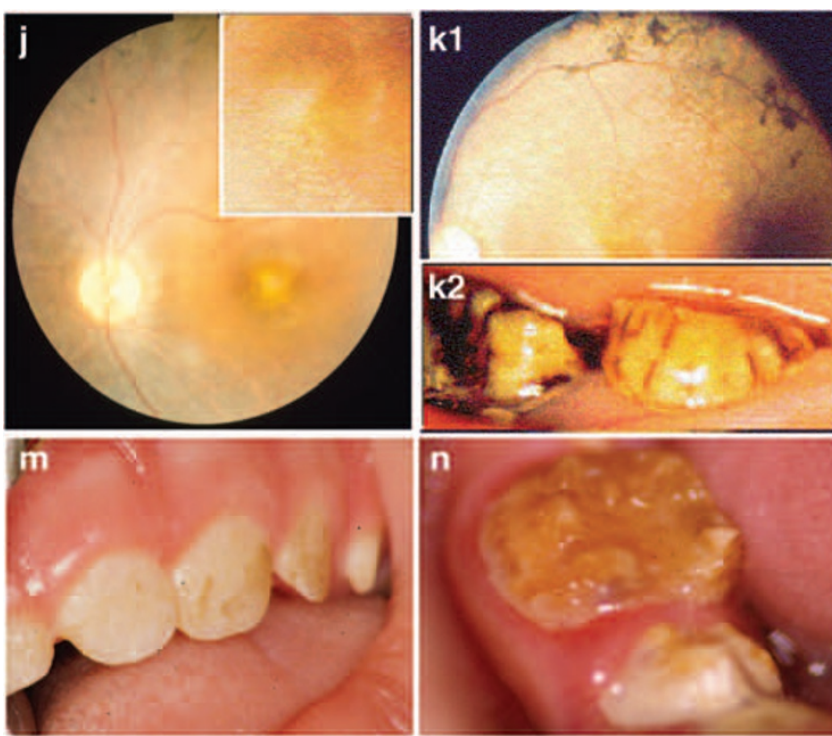
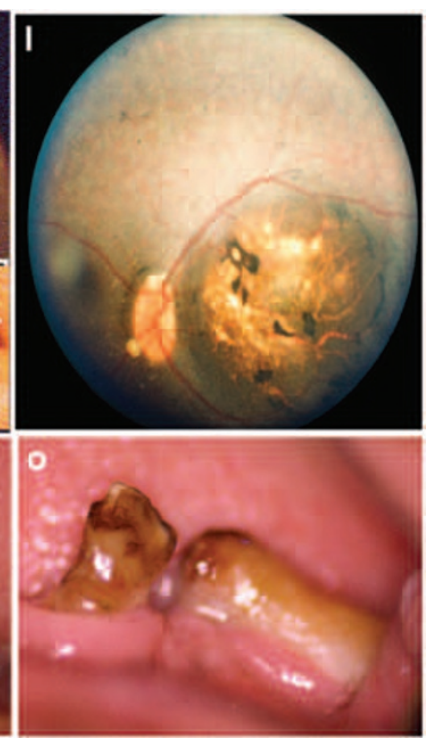

Figure 2 Type B CRD and AI (Jalili syndrome). Upper row (a, b): fundus photographs and angiogram of the 10-year-old sibling; (c) 5-year-old youngest sister with phenotype B Jalili syndrome showing normal-looking macula and absence of peripheral pigmentary proliferation. Second row (d-f): AI in the 10-, 6-, and 5-year-old siblings, respectively; (d) staining and calculus formation in the upper teeth, note pitting from nut cracking, and irregular dentition in the lower teeth; (e) marked calculus formation and AOB in the 6-yearold sister; (f) AOB without staining or calculus formation in the youngest sister. Third row (g-i): evolution of macular lesions in phenotype A (Gaza A); (g) bull's eye lesion becoming more confluent with eventual chorioretinal atrophy and excavation (enlarged in the inset). Fourth row $(j, k)$ : retina in members of the Gaza A genetic family from different sibship from a different patrilineal descent; (j) flat atrophic macular degeneration, (inset) atrophic macular degeneration from a different sibling; (k1) peripheral pigmentary clumping; ( $\mathrm{k} 2$ ) slightly different AI morphology in siblings of this sibship of type A; (l) macular coloboma (posterior staphyloma) of sibling $\mathrm{C}$, note the remnant of posterior hyaloid artery at 8 o'clock position, which was absent in his parents and siblings. Fifth row $(\mathrm{m}-\mathrm{o})$ : evolution of dental lesions in $\mathrm{AI} ;(\mathrm{m})$ teeth are initially pitted; (n) further loss of enamel and staining; (o) eventual loss of teeth in a 50-year-old patient from Gaza A. 